\title{
Qual é a expressão correta para o trabalho realizado pela força de atrito cinético?
}

\author{
What is the correct expression for the work done by the force of kinetic friction? \\ Paulo Peixoto*10 \\ ${ }^{1}$ Universidade Federal de Pernambuco, Núcleo de Formação Docente, Caruaru, PE, Brasil
}

Recebido em 25 de Maio, 2018. Revisado em 09 de Julho, 2018. Aceito em 09 de Julho, 2018.

\begin{abstract}
Neste artigo, argumento que a expressão matemática correta para o trabalho realizado pela força de atrito cinético depende de como o corpo que sofre a ação do atrito está sendo modelado: como uma partícula ou como um sistema de partículas. Defendo que ambas as alternativas são válidas, e que a escolha entre uma e outra depende do tipo de informação que nos interessa, no problema. No segundo caso, contudo, a própria expressão "trabalho realizado pela força de atrito cinético" precisa ser modificada.

Palavras-chave: força de atrito cinético, trabalho, teoremas trabalho - energia cinética, sistemas de partículas, modelagem.

In this paper, I argue that the correct mathematical expression for the work done by the force of kinetic friction depends on how the body who is subject to friction is being modelled: as a particle or as a system of particles. I argue that both alternatives are valid, and that the choice of one of them depends on the type of information that interests us in the problem. In the second case, however, the very expression "work done by the force of kinetic friction" needs to be modified.
\end{abstract}

Keywords: force of kinetic friction, work, work - kinetic energy theorems, systems of particles, modelling.

\section{Introdução}

A Fig.1 1 ilustra um bloco de massa $M$ movendo-se com velocidade $\mathbf{v}$ sobre uma superfície horizontal. Há atrito entre o bloco e a superfície, e, portanto, o módulo de $\mathbf{v}$ diminui com o tempo. Qual é o trabalho realizado pela força de atrito cinético, $\mathbf{f}_{\mathbf{c}}$, ao longo de um deslocamento $d$ sofrido pelo bloco?

A resposta usual para esta pergunta é: $-f_{c} d$, em que $f_{\mathrm{c}}$ é o módulo de $\mathbf{f}_{\mathbf{c}}$. Contudo, em um trabalho publicado em 2011 [1], Nelson e Carneiro Filho afirmaram que tal expressão está incorreta, e propuseram uma outra (que discutiremos mais adiante) - supostamente $a$ expressão correta.

Discordando da conclusão rígida desses autores, neste artigo argumento que a resposta para a questão acima depende, entre outras coisas, de como o corpo que sofre a ação do atrito cinético está sendo modelado: como uma partícula ou como um sistema de partículas. Defendo que ambas as alternativas de modelagem são válidas, e que a escolha entre uma e outra depende do tipo de informação que nos interessa, no problema. No caso da modelagem do corpo como um sistema de partículas, porém, argumento que a expressão "trabalho realizado pela força de atrito cinético" é inadequada, e proponho uma outra expressão.

*Endereço de correspondência: phrpeixoto@yahoo.com.br

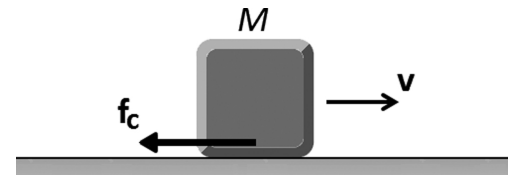

Figura 1: Bloco desacelerando, devido ao atrito cinético com o piso.

\section{Teoremas trabalho - energia cinética}

Nesta seção, trabalharemos a fundamentação teórica necessária à realização das modelagens apresentadas nas seções 3 e 4 .

Segundo o teorema trabalho - energia cinética para uma partícula de massa $m$,

$$
W_{\text {total }}=\Delta T(\text { para uma partícula })
$$

em que $\Delta T$ é a variação da energia cinética da partícula entre dois estados 1 e 2 e $W_{\text {total }}$ é a soma dos trabalhos de todas as forças $\mathbf{F}_{\mathbf{1}}, \mathbf{F}_{\mathbf{2}}, \ldots, \mathbf{F}_{\mathbf{n}}$ que atuam sobre a partícula na evolução do estado 1 ao estado 2. Será útil revisarmos a demonstração deste teorema. Temos:

$$
W_{\text {total }}=\int_{\Gamma} \mathbf{F}_{\mathbf{1}} \cdot \mathrm{d} \mathbf{r}+\int_{\Gamma} \mathbf{F}_{\mathbf{2}} \cdot \mathrm{d} \mathbf{r}+\cdots+\int_{\Gamma} \mathbf{F}_{\mathbf{n}} \cdot \mathrm{d} \mathbf{r},
$$


em que $\Gamma$ é a trajetória da partícula entre os estados 1 e 2, e r é seu vetor posição, no referencial do laboratório. Usando uma propriedade básica do cálculo integral, podemos reescrever a igualdade 2 como

$$
W_{\text {total }}=\int_{\Gamma} \mathbf{F}_{\text {res }} \cdot \mathrm{d} \mathbf{r},
$$

em que $\mathbf{F}_{\text {res }}=\mathbf{F}_{\mathbf{1}}+\mathbf{F}_{\mathbf{2}}+\cdots+\mathbf{F}_{\mathbf{n}}$ é a força resultante sobre a partícula. Podemos então afirmar que, no caso de uma partícula única, o trabalho total é igual ao trabalho da força resultante. Fazendo uso, na igualdade (3), da segunda lei de Newton na forma $\mathbf{F}_{\mathbf{r e s}}=m \mathrm{~d} \mathbf{v} / \mathrm{d} t$, em que $\mathbf{v}=\mathrm{d} \mathbf{r} / \mathrm{d} t$, e observando que $\mathbf{v} \cdot \mathrm{d} \mathbf{v}=v \mathrm{~d} v{ }^{1}$ (em que $v=|\mathbf{v}|)$, obtemos $W_{\text {total }}=\Delta\left(m v^{2} / 2\right)$. Com a definição $T \equiv m v^{2} / 2$ para a energia cinética $T$ de uma partícula de massa $m$ com velocidade de módulo $v$, segue a igualdade (1).

Passemos ao teorema trabalho - energia cinética para um sistema de partículas de massa total $M$. Definindo, por extensão, $W_{\text {total }}$ como a soma dos trabalhos de todas as forças (internas e externas) que atuam sobre as partículas do sistema na evolução de um estado 1 a um estado 2 do mesmo, e reunindo em uma só integral à semelhança do que fizemos da igualdade (2) para a igualdade (3) - os trabalhos de todas as forças (internas e externas) que atuam em uma determinada partícula, obtemos:

$$
\begin{aligned}
W_{\text {total }} & =\int_{\Gamma_{1}} \mathbf{F}_{\text {res }_{1}} \cdot \mathrm{d} \mathbf{r}_{1}+\int_{\Gamma_{2}} \mathbf{F}_{\text {res }_{2}} \cdot \mathrm{d} \mathbf{r}_{2}+\cdots \\
& +\int_{\Gamma_{N}} \mathbf{F}_{\text {res }_{\mathbf{N}}} \cdot \mathrm{d} \mathbf{r}_{\mathbf{N}}
\end{aligned}
$$

em que $\mathbf{F}_{\text {res }}$ ( $(i \in\{1, \ldots, N\})$ é a força resultante sobre a i-ésima partícula do sistema (que pode envolver forças internas e externas), $\Gamma_{i}$ é a trajetória de tal partícula na evolução do estado 1 ao estado 2 do sistema, e $\mathbf{r}_{\mathbf{i}}$ é seu vetor posição, no referencial do laboratório. Fazendo uso, na igualdade (4), para cada partícula, da segunda lei de Newton na forma $\mathbf{F}_{\mathbf{r e s}_{\mathbf{i}}}=m_{i} \mathrm{~d} \mathbf{v}_{\mathbf{i}} / \mathrm{d} t$ (em que $m_{i}$ e $\mathbf{v}_{\mathbf{i}}$ são, respectivamente, a massa e a velocidade, no referencial do laboratório, da i-ésima partícula do sistema), após alguns cálculos simples, já familiares, podemos concluir que o trabalho total sobre o sistema, na evolução de um estado 1 a um estado 2 do mesmo, é igual à soma das variações das energias cinéticas de suas partículas. Definindo a energia cinética de um sistema como a soma das energias cinéticas das partículas que o constituem (ou seja, $T=\sum_{i=1}^{N} \frac{1}{2} m_{i} v_{i}^{2}$ ), concluímos que o trabalho total sobre um sistema de partículas é igual à variação da energia cinética do sistema, entre dois estados do mesmo - e este é o teorema trabalho - energia cinética para um sistema de partículas. Note que este teorema também pode ser expresso através da igualdade

$$
W_{\text {total }}=\Delta T(\text { para um sistema }),
$$

${ }^{1}$ Para obter esta igualdade, projete dv na direção de $\mathbf{v}$, e observe que tal projeção é igual à variação infinitesimal correspondente $\mathrm{d} v$ do módulo de $\mathbf{v}$. mas sendo, agora, $\Delta T$ a variação da energia cinética do sistema entre dois estados 1 e 2 , e $W_{\text {total a soma dos }}$ trabalhos de todas as forças (internas e externas) que atuam sobre as partículas do sistema na evolução do estado 1 ao estado 2.

A energia cinética de um sistema de partículas de massa total $M$ pode ser convenientemente expressa como $T=\frac{1}{2} M v_{\mathrm{cm}}^{2}+T_{\mathrm{rel}}$, em que $v_{\mathrm{cm}}$ é o módulo da velocidade do centro de massa do sistema (no referencial do laboratório) e $T_{\text {rel }}$ é a soma das energias cinéticas das partículas do sistema relativamente ao centro de massa (ou seja, no referencial do centro de massa). Para obtermos este resultado, basta substituirmos, em $T=\sum_{i=1}^{N} \frac{1}{2} m_{i} v i^{2}=\sum_{i=1}^{N} \frac{1}{2} m_{i}\left(\mathbf{v}_{\mathbf{i}} \cdot \mathbf{v}_{\mathbf{i}}\right)$, a velocidade $\mathbf{v}_{\mathbf{i}}$ da i-ésima partícula do sistema por $\mathbf{v}_{\mathbf{c m}}+\mathbf{v}_{\mathbf{i}, \mathbf{c m}}$, em que $\mathbf{v}_{\mathbf{c m}}$ é a velocidade do centro de massa do sistema (no referencial do laboratório) e $\mathbf{v}_{\mathbf{i}, \mathbf{c m}}$ é a velocidade da i-ésima partícula em relação ao centro de massa. $2^{2}$ Com tal expressão para $T$, podemos reescrever a igualdade (5) como

$$
W_{\text {total }}=\Delta\left(\frac{1}{2} M v_{\mathrm{cm}}^{2}\right)+\Delta T_{\text {rel }},
$$

em que $T_{\text {rel }}=\sum_{i=1}^{N} \frac{1}{2} m_{i} v_{i, \mathrm{~cm}}^{2}$.

Obteremos, a seguir, o segundo membro da igualdade (6) diretamente da igualdade (4) - ou seja, sem passarmos previamente pela igualdade (5). Curiosamente, trata-se de um caminho um pouco mais trabalhoso, e com algumas sutilezas, mas que constituirá o núcleo da fundamentação teórica necessária à realização das modelagens apresentadas nas seções 3 e 4, relativas ao problema do cálculo do trabalho realizado pela força de atrito cinético. Portanto, sugiro que você dê especial atenção a essa parte.

Substituindo, em (4), $\mathbf{r}_{\mathbf{i}}$ por $\mathbf{r}_{\mathbf{c m}}+\mathbf{r}_{\mathbf{i}, \mathbf{c m}}$, em que $\mathbf{r}_{\mathbf{c m}}$ é o vetor posição do centro de massa do sistema (no referencial do laboratório) e $\mathbf{r}_{\mathbf{i}, \mathbf{c m}}$ é o vetor posição da i-ésima partícula em relação ao centro de massa, obtemos:

$$
\begin{aligned}
W_{\mathrm{total}} & =\int_{\Gamma_{\mathrm{cm}}} \mathbf{F}_{\mathbf{r e s}_{\mathbf{1}}} \cdot \mathrm{d} \mathbf{r}_{\mathbf{c m}}+\int_{\Gamma_{1, \mathrm{~cm}}} \mathbf{F}_{\mathbf{r e s}_{\mathbf{1}}} \cdot \mathrm{d} \mathbf{r}_{\mathbf{1}, \mathbf{c m}} \\
& +\int_{\Gamma_{\mathrm{cm}}} \mathbf{F}_{\mathbf{r e s}_{\mathbf{2}}} \cdot \mathrm{d} \mathbf{r}_{\mathbf{c m}}+\int_{\Gamma_{2, \mathrm{~cm}}} \mathbf{F}_{\mathbf{r e s}_{\mathbf{2}}} \cdot \mathrm{d} \mathbf{r}_{\mathbf{2}, \mathbf{c m}} \\
& \vdots \\
& +\int_{\Gamma_{\mathrm{cm}}} \mathbf{F}_{\mathbf{r e s}_{\mathbf{N}}} \cdot \mathrm{d} \mathbf{r}_{\mathbf{c m}}+\int_{\Gamma_{N, \mathrm{~cm}}} \mathbf{F}_{\mathbf{r e s}_{\mathbf{N}}} \cdot \mathrm{d} \mathbf{r}_{\mathbf{N}, \mathbf{c m}}
\end{aligned}
$$

em que $\Gamma_{\mathrm{cm}}$ é a trajetória do centro de massa (no referencial do laboratório) e $\Gamma_{i, \mathrm{~cm}}$ é a trajetória da i-ésima partícula, no referencial do centro de massa, na evolução do estado 1 ao estado 2 do sistema. Reunindo em uma única integral o primeiro termo de cada linha em (7),

\footnotetext{
2Em uma parte dos cálculos, obtemos a expressão $\mathbf{v}_{\mathbf{c m}} \cdot \sum_{i=1}^{N} m_{i} \mathbf{v}_{\mathbf{i}, \mathbf{c m}}$, e devemos observar que $\sum_{i=1}^{N} m_{i} \mathbf{v}_{\mathbf{i}, \mathbf{c m}}=$ $M \mathbf{v}_{\mathbf{c m}, \mathbf{c m}}=0$, pois a velocidade do centro de massa no referencial do próprio centro de massa é, obviamente, nula.
} 
obtemos:

$$
\begin{aligned}
W_{\text {total }} & =\int_{\Gamma_{\mathrm{cm}}} \mathbf{F}_{\mathbf{r e s}}^{\mathbf{e x t}} \cdot \mathrm{d} \mathbf{r}_{\mathbf{c m}} \\
& +\int_{\Gamma_{1, \mathrm{~cm}}} \mathbf{F}_{\mathbf{r e s}_{1}} \cdot \mathrm{d} \mathbf{r}_{1, \mathbf{c m}}+\int_{\Gamma_{2, \mathrm{~cm}}} \mathbf{F}_{\mathbf{r e s}_{\mathbf{2}}} \cdot \mathrm{d} \mathbf{r}_{\mathbf{2}, \mathbf{c m}} \\
& +\cdots+\int_{\Gamma_{N, \mathrm{~cm}}} \mathbf{F}_{\mathbf{r e s}_{\mathbf{N}}} \cdot \mathrm{d} \mathbf{r}_{\mathbf{N}, \mathbf{c m}},
\end{aligned}
$$

em que $\mathbf{F}_{\text {res }}^{\text {ext }}=\mathbf{F}_{\text {res }_{1}}+\mathbf{F}_{\text {res }_{2}}+\cdots+\mathbf{F}_{\text {res }_{\mathbf{N}}}$ é a resultante das forças externas sobre o sistema. ${ }^{3}$ Trabalhando com a segunda lei de Newton para um sistema de partículas de massa total $M, \mathbf{F}_{\text {res }}^{\mathbf{e x t}}=M \mathrm{~d} \mathbf{v}_{\mathbf{c m}} / \mathrm{d} t$, a primeira integral em (8) nos fornece o primeiro termo do segundo membro em (6):

$$
\int_{\Gamma_{\mathrm{cm}}} \mathbf{F}_{\mathbf{r e s}}^{\mathbf{e x t}} \cdot \mathrm{d} \mathbf{r}_{\mathbf{c m}}=\Delta\left(\frac{1}{2} M v_{\mathrm{cm}}^{2}\right) .
$$

Veremos que as demais integrais em (8) resultam no segundo termo do segundo membro em $(\sqrt{6})$ - o que concluirá nossa demonstração da igualdade (6), partindo da igualdade (4). Ou seja, para concluir a demonstração, vamos mostrar que

$$
\sum_{i=1}^{N} \int_{\Gamma_{i, \mathrm{~cm}}} \mathbf{F}_{\mathbf{r e s}_{\mathbf{i}}} \cdot \mathrm{d} \mathbf{r}_{\mathbf{i}, \mathbf{c m}}=\Delta T_{\mathrm{rel}} .
$$

Em primeiro lugar, devemos atentar para o seguinte: $\mathbf{F}_{\text {resi }_{\mathbf{i}}}$ é a força resultante sobre a i-ésima partícula no referencial do laboratório, não no referencial do centro de massa. Portanto, em geral temos $\mathbf{F}_{\text {res }_{\mathbf{i}}} \neq \mathbf{F}_{\mathbf{r e s}_{\mathbf{i}}, \mathbf{c m}}$, e, consequentemente,

$$
\int_{\Gamma_{i, \mathrm{~cm}}} \mathbf{F}_{\mathbf{r e s}_{\mathbf{i}}} \cdot \mathrm{d} \mathbf{r}_{\mathbf{i}, \mathbf{c m}} \neq \int_{\Gamma_{i, \mathrm{~cm}}} \mathbf{F}_{\mathbf{r e s}_{\mathbf{i}}, \mathbf{c m}} \cdot \mathrm{d} \mathbf{r}_{\mathbf{i}, \mathbf{c m}}
$$

Esta desigualdade torna-se uma igualdade no caso particular em que o centro de massa se move com velocidade constante no referencial do laboratório (pois, assim, trabalhando com a transformação de Galileu obte$\operatorname{mos} \mathrm{d} \mathbf{v}_{\mathbf{i}, \mathbf{c m}} / \mathrm{d} t=\mathrm{d}\left(\mathbf{v}_{\mathbf{i}}-\mathbf{v}_{\mathbf{c m}}\right) / \mathrm{d} t=\mathrm{d} \mathbf{v}_{\mathbf{i}} / \mathrm{d} t$, e, portanto, $\left.\mathbf{F}_{\mathbf{r e s}_{\mathbf{i}}}=m_{i} \mathrm{~d} \mathbf{v}_{\mathbf{i}} / \mathrm{d} t=m_{i} \mathrm{~d} \mathbf{v}_{\mathbf{i}, \mathbf{c m}} / \mathrm{d} t=\mathbf{F}_{\mathbf{r e s}_{\mathbf{i}}, \mathbf{c m}}\right)$. Nesse caso particular, as contas ficam mais fáceis, pois seguindo um desenvolvimento semelhante ao realizado na demonstração da igualdade (1) obtemos (já somando sobre todas as partículas):

$$
\begin{gathered}
\sum_{i=1}^{N} \int_{\Gamma_{i, \mathrm{~cm}}} \mathbf{F}_{\mathbf{r e s}_{\mathbf{i}}, \mathbf{c m}} \cdot \mathrm{d} \mathbf{r}_{\mathbf{i}, \mathbf{c m}} \\
=\sum_{i=1}^{N} \int_{\Gamma_{i, \mathrm{~cm}}}\left(m_{i} \mathrm{~d} \mathbf{v}_{\mathbf{i}, \mathbf{c m}} / \mathrm{d} t\right) \cdot \mathrm{d} \mathbf{r}_{\mathbf{i}, \mathbf{c m}} \\
=\sum_{i=1}^{N} \int_{\Gamma_{i, \mathrm{~cm}}} m_{i} \mathbf{v}_{\mathbf{i}, \mathbf{c m}} \cdot \mathrm{d} \mathbf{v}_{\mathbf{i}, \mathbf{c m}} \\
=\sum_{i=1}^{N} \int_{\Gamma_{i, \mathrm{~cm}}} m_{i} v_{i, \mathrm{~cm}} \mathrm{~d} v_{i, \mathrm{~cm}}=\sum_{i=1}^{N} \Delta\left(m_{i} v_{i, \mathrm{~cm}}^{2} / 2\right) \\
=\Delta\left(\sum_{i=1}^{N} m_{i} v_{i, \mathrm{~cm}}^{2} / 2\right)=\Delta T_{\mathrm{rel}} .
\end{gathered}
$$

${ }^{3}$ Cada uma das forças resultantes $\mathbf{F}_{\mathbf{r e s}_{1}}, \mathbf{F}_{\mathbf{r e s}_{2}}, \ldots, \mathbf{F}_{\mathbf{r e s}_{\mathbf{N}}}$ pode envolver forças internas e externas, mas, na soma $\mathbf{F}_{\mathbf{r e s}_{1}}+\mathbf{F}_{\mathbf{r e s}_{2}}+$ $\cdots+\mathbf{F}_{\text {res }_{\mathbf{N}}}$, obtemos a resultante das forças externas porque a resultante das forças internas é nula (uma consequência da terceira lei de Newton).
Temos em (11), portanto, a demonstração da igualdade (10) para o caso particular em que o centro de massa se move com velocidade constante no referencial do laboratório, e, portanto, $\mathbf{F}_{\mathbf{r e s}_{\mathbf{i}}}=\mathbf{F}_{\mathbf{r e s}_{\mathbf{i}}, \mathbf{c m}}$.

Mas não precisamos dessa restrição. No caso geral, temos:

$$
\begin{gathered}
\sum_{i=1}^{N} \int_{\Gamma_{i, \mathrm{~cm}}} \mathbf{F}_{\mathbf{r e s}_{\mathbf{i}}} \cdot \mathrm{d} \mathbf{r}_{\mathbf{i}, \mathbf{c m}} \\
=\sum_{i=1}^{N} \int_{\Gamma_{i, \mathrm{~cm}}}\left(m_{i} \mathrm{~d} \mathbf{v}_{\mathbf{i}} / \mathrm{d} t\right) \cdot \mathrm{d} \mathbf{r}_{\mathbf{i}, \mathbf{c m}} \\
=\sum_{i=1}^{N} \int_{\Gamma_{i, \mathrm{~cm}}} m_{i} \mathbf{v}_{\mathbf{i}, \mathbf{c m}} \cdot \mathrm{d} \mathbf{v}_{\mathbf{i}} \\
=\sum_{i=1}^{N} \int_{\Gamma_{i, \mathrm{~cm}}} m_{i} \mathbf{v}_{\mathbf{i}, \mathbf{c m}} \cdot \mathrm{d}\left(\mathbf{v}_{\mathbf{c m}}+\mathbf{v}_{\mathbf{i}, \mathbf{c m}}\right) \\
=\sum_{i=1}^{N} \int_{\Gamma_{i, \mathrm{~cm}}} m_{i} \mathbf{v}_{\mathbf{i}, \mathbf{c m}} \cdot \mathrm{d} \mathbf{v}_{\mathbf{c m}} \\
+\sum_{i=1}^{N} \int_{\Gamma_{i, \mathrm{~cm}}} m_{i} \mathbf{v}_{\mathbf{i}, \mathbf{c m}} \cdot \mathrm{d} \mathbf{v}_{\mathbf{i}, \mathbf{c m}} .
\end{gathered}
$$

Mostramos, em (11), que o último destes somatórios resulta em $\Delta T_{\text {rel }}$. Portanto, para concluir a demonstração da igualdade (10) precisamos mostrar que o penúltimo dos somatórios em (12) é nulo; ou seja, precisamos mostrar que

$$
\sum_{i=1}^{N} \int_{\Gamma_{i, \mathrm{~cm}}} m_{i} \mathbf{v}_{\mathbf{i}, \mathbf{c m}} \cdot \mathrm{d} \mathbf{v}_{\mathbf{c m}}=0 .
$$

$\mathrm{Na}$ evolução de um determinado estado 1 a um determinado estado 2 do sistema, cada partícula segue seu caminho $\Gamma_{i, \mathrm{~cm}}, i \in\{1,2, \ldots, N\}$ (e da combinação (ponderada) desses caminhos resulta a trajetória do centro de massa). Em princípio, isso impede a permutação das posições de " $\sum_{i=1}^{N}$ " e " $\int_{\Gamma_{i, \mathrm{~cm}}}$ " em 13 . Mas, para resolvermos este problema, basta observarmos que as $N$ variáveis $\mathbf{v}_{\mathbf{i}, \mathbf{c m}}$, e também $\mathbf{v}_{\mathbf{c m}}$, são, todas, funções do tempo $(t)$, e que, portanto, podemos reescrever:

$$
\sum_{i=1}^{N} \int_{\Gamma_{i, \mathrm{~cm}}} m_{i} \mathbf{v}_{\mathbf{i}, \mathbf{c m}} \cdot \mathrm{d} \mathbf{v}_{\mathbf{c m}}=\sum_{i=1}^{N} \int_{t_{1}}^{t_{2}} m_{i} \mathbf{v}_{\mathbf{i}, \mathbf{c m}}(t) \cdot \mathrm{d} \mathbf{v}_{\mathbf{c m}}(t) .
$$

Agora sim, podemos permutar as posições dos sinais de somatório e de integração, obtendo:

$$
\sum_{i=1}^{N} \int_{\Gamma_{i, \mathrm{~cm}}} m_{i} \mathbf{v}_{\mathbf{i}, \mathbf{c m}} \cdot \mathrm{d} \mathbf{v}_{\mathbf{c m}}=\int_{t=t_{1}}^{t=t_{2}}\left(\sum_{i=1}^{N} m_{i} \mathbf{v}_{\mathbf{i}, \mathbf{c m}}\right) \cdot \mathrm{d} \mathbf{v}_{\mathbf{c m}} .
$$

A expressão entre parênteses, em (14), é igual a $M \mathbf{v}_{\mathbf{c m}, \mathbf{c m}}$ e como $\mathbf{v}_{\mathbf{c m}, \mathbf{c m}}=0$, segue a igualdade $(13)-$ o que conclui nossa demonstração da igualdade (6), partindo da igualdade (4)

Recapitulando, no início desta seção revisamos o teorema trabalho - energia cinética para uma partícula de massa $m$ e para um sistema de partículas de massa total $M$. Em seguida, mostramos que a variação da energia cinética do sistema pode ser convenientemente expressa na forma que constitui o segundo membro da igualdade (6). Na sequência, deduzimos tal expressão diretamente da igualdade (4) - etapa particularmente importante para o que vem a seguir, nas seções 3 e 4 . Serão de particular interesse as igualdades (9) e 10$)$. 
Antes de encerrarmos esta seção, convém enfatizar que, diferentemente do caso de uma partícula única, para um sistema de partículas não podemos afirmar que o trabalho total é igual ao trabalho da força resultante sobre o sistema (pois, na igualdade (8), o segundo membro não se encerra na primeira integral). No caso de uma partícula única, foi possível passarmos da igualdade (2) para a igualdade (3) porque todas as integrais em (2) envolvem um mesmo caminho $\Gamma$ - afinal, trata-se de uma única partícula. Contudo, trabalhando com um sistema de $N$ partículas, em geral temos, na evolução de um estado 1 a um estado 2 do sistema, $N$ caminhos distintos $\Gamma_{1}, \Gamma_{2}, \ldots, \Gamma_{N}$ (ou $\Gamma_{1, \mathrm{~cm}}, \Gamma_{2, \mathrm{~cm}}, \ldots, \Gamma_{N, \mathrm{~cm}}$, no referencial do centro de massa), um para cada partícula - daí a presença das $N$ últimas integrais em (8), e, portanto, a impossibilidade de obtenção de uma igualdade semelhante à igualdade (3).

Estamos prontos para discutir a modelagem do bloco da Fig. 1 como uma partícula e como um sistema de partículas, começando por esta.

\section{Modelando o bloco da Fig. 1 como um sistema de partículas}

Na mecânica clássica, uma partícula é algo como um corpo, com massa, mas de "tamanho nulo" (um ponto, no sentido geométrico), ou com dimensões infinitesimais (como adequado para a integração de um volume finito). Trata-se de uma abstração, que leva a um desenvolvimento sólido e rigoroso da teoria a partir de uma fundação relativamente simples. Para estudar o movimento de corpos reais, como o bloco representado na Fig.1. podemos modelá-los como um sistema de partículas. Se o corpo gira, vibra, ou de algum modo se deforma, dizemos que é devido ao movimento das partículas que o constituem. Agora, esteja atento(a): por "partículas" não estamos nos referindo aqui, por exemplo, a elétrons, prótons e nêutrons. A mecânica clássica não descreve adequadamente a natureza nessas dimensões. Quando, trabalhando com a mecânica clássica, descrevemos um corpo macroscópico como um sistema de partículas, tenha em mente que estamos imaginando-o sendo formado (ou melhor, estamos modelando-o como sendo formado) por um certo número $N$ de partículas "clássicas" - ou seja, que obedecem às leis da mecânica clássica. Mesmo em uma visão realista de mundo $4^{4}$ não se trata de suas reais partículas constituintes, mas de uma abstração - que só se justifica conforme as previsões teóricas do modelo se harmonizem com resultados experimentais. Portanto, perceba que não é apenas modelando o bloco da Fig.11 como uma partícula que introduzimos simplificações em nosso desenvolvimento; também o fazemos modelando-o como um sistema de partículas clássicas. O que quero

\footnotetext{
${ }^{4}$ Em termos simples, o realista científico é aquele que defende que ao menos algumas das entidades postuladas pela ciência, como elétrons, fótons, quarks etc., realmente existem. [O autor deste artigo há muitos anos adotou a visão de que a ciência busca harmonia entre imagens de mundo (modelos, teorias,...) e experiências de mundo (experimentos, observações,...), mas não é capaz de penetrar na essência das coisas.]
}

dizer com isso é que não dá para reclamar uma abordagem como absolutamente correta, e a outra como errada; como veremos, a escolha depende do tipo de informação que nos interessa, no problema.

Feitas essas considerações, passemos à modelagem.

Logo de início, veremos que, optando por modelar o bloco da Fig. 1 como um sistema de partículas, a expressão "trabalho realizado pela força de atrito cinético, $\mathbf{f}_{\mathbf{c}}$, ao longo de um deslocamento $d$ sofrido pelo bloco" é inadequada.

Quando dizemos que o peso do bloco da Fig.11é $\mathbf{P}=$ $M \mathbf{g}$, em que $M$ é a massa do bloco e $\mathbf{g}$ é o vetor aceleração da gravidade local (desprezemos as ínfimas variações de $\mathbf{g}$ com a altitude, ao longo do bloco), estamos nos referindo à soma vetorial dos pesos de todas as $N$ partículas que o constituem (na modelagem do bloco como um sistema de partículas). Sendo $m_{i}$ a massa da i-ésima partícula, temos $\mathbf{P}=\sum_{i=1}^{N} \mathbf{P}_{\mathbf{i}}=\sum_{i=1}^{N}\left(m_{i} \mathbf{g}\right)=\left(\sum_{i=1}^{N} m_{i}\right) \mathbf{g}=M \mathbf{g}, M=$ $\sum_{i=1}^{N} m_{i}$. De modo análogo, o que chamamos de força

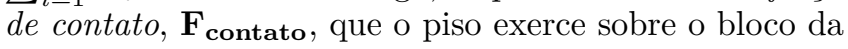
Fig. 1. é a soma vetorial de todas as forças exercidas pelo piso sobre partículas do bloco. E o que chamamos de força normal, $\mathbf{f}_{\mathbf{n}}$, e de força de atrito cinético, $\mathbf{f}_{\mathbf{c}}$, são as componentes de $\mathbf{F}_{\text {contato }}$ perpendicular e paralela ao plano de contato, respectivamente. Pois bem, se estamos interessados em calcular o trabalho realizado pela força de atrito cinético, $\mathbf{f}_{\mathbf{c}}$, ao longo de um deslocamento $d$ sofrido pelo bloco, nos deparamos com este problema: $\mathbf{f}_{\mathbf{c}}$ não constitui uma força, mas uma soma de forças (ou, mais precisamente, a componente paralela ao plano de contato da soma de forças $\mathbf{F}_{\text {contato }}$ ). Assim, modelando o bloco da Fig.1 como um sistema de partículas, por enquanto proponho substituir a pergunta apresentada no início da primeira seção pela seguinte: Qual é o trabalho total realizado pelas forças que o piso exerce sobre partículas do bloco da Fig. 1. ao longo de um deslocamento d de seu centro de massa? Chamaremos esse trabalho de trabalho do contato, $W_{\text {contato }}$.

Como não há informação para modelarmos individualmente as forças exercidas pelo piso, nem as trajetórias das partículas do bloco sujeitas a tais forças (ao longo de um deslocamento $d$ do centro de massa do bloco da Fig. 1), o que temos para o cálculo de $W_{\text {contato são as }}$ igualdades (8), (9) e (10).

Considerando que as únicas forças externas sobre o bloco da Fig.11 são as forças exercidas pelo piso, além dos pesos das $N$ partículas que o constituem, podemos escrever:

$$
W_{\text {total }}=W_{\text {contato }}+W_{\text {pesos }}+W_{\mathrm{F}_{\text {internas }}},
$$

em que $W_{\text {pesos }}$ é a soma dos trabalhos realizados pelos pesos das $N$ partículas que constituem o bloco e $W_{\mathrm{F}_{\text {internas }}}$ é a soma dos trabalhos das forças internas ao bloco - ou seja, das forças exercidas pelas partículas que constituem o bloco, umas sobre as outras. Segue então que

$$
W_{\text {contato }}=W_{\text {total }}-W_{\text {pesos }}-W_{\mathrm{F}_{\text {internas }}} .
$$

Vamos nos ocupar, agora, com cada um dos termos do segundo membro da igualdade (15), começando com 
$W_{\text {pesos. }}$ O trabalho realizado pelo peso da i-ésima partícula do bloco é $W_{P_{i}}=-m_{i} g \Delta h_{i}$, em que $\Delta h_{i}$ é a variação de altura de tal partícula após o deslocamento $d$ do centro de massa do bloco. Segue que $W_{\text {pesos }}=\sum_{i=1}^{N} W_{P_{i}}=$ $\sum_{i=1}^{N}-m_{i} g \Delta h_{i}=-g \Delta\left(\sum_{i=1}^{N} m_{i} h_{i}\right)=-g \Delta\left(M h_{\mathrm{cm}}\right)=$ $-M g \Delta h_{\mathrm{cm}}$, em que $\Delta h_{\mathrm{cm}}$ é a variação de altura do centro de massa do bloco da Fig. 1] após o deslocamento (horizontal) $d$ desse centro de massa. Assim, mesmo havendo, após tal deslocamento horizontal, pequenas variações de altura para as $N$ partículas que constituem o bloco, a altura de seu centro de massa permanece a mesma, e, portanto,

$$
W_{\text {pesos }}=0 .
$$

O cálculo de $W_{\mathrm{F}_{\text {internas }}}$ em princípio é um problema. Afinal, que elementos temos para realizar - mesmo com um modelo simplificado - um cálculo direto desse trabalho? Conseguimos, no entanto, contornar essa dificuldade considerando que, além de podermos modelar o bloco da Fig.1 (para o cálculo de $W_{\text {contato }}$ ) como um sistema de $N$ partículas clássicas, as forças internas são tais que $W_{\mathrm{F}_{\text {internas }}}$ só depende das posições finais e iniciais das $N$ partículas, no referencial do centro de massa. Com essa hipótese, podemos introduzir uma função energia potencial $U_{\text {int }}$ - uma função das posições das $N$ partículas, no referencial do centro de massa - tal qu ${ }^{5}$

$$
W_{\mathrm{F}_{\text {internas }}}=-\Delta U_{\mathrm{int}} \text {. }
$$

O que falta, agora, é uma expressão para $W_{\text {total }}$ À primeira vista, poderíamos usar a igualdade (6) (ou, equivalentemente, as igualdades (8), (9) e (10), conjuntamente), mas ela não nos seria muito útil, porque a

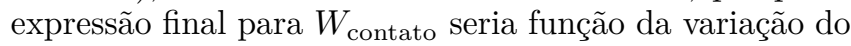
quadrado da velocidade $v_{\mathrm{cm}}$ do centro de massa do bloco da Fig.11 após um deslocamento $d$ desse centro de massa. O que queremos é que apareça " $f_{\mathrm{c}}$ " na expressão final para $W_{\text {contato }}$ Assim, faremos uso, em (8), da igualdade (10), mas não da igualdade (9), obtendo:

$$
W_{\text {total }}=\int_{\Gamma_{\mathrm{cm}}} \mathbf{F}_{\text {rest }}^{\text {ext }} \cdot \mathrm{d} \mathbf{r}_{\mathbf{c m}}+\Delta T_{\mathrm{rel}} .
$$

Como o centro de massa do bloco se move paralelamente ao piso, a integral na igualdade acima fica:

$$
\begin{aligned}
\int_{\Gamma_{\mathrm{cm}}} \mathbf{F}_{\mathbf{r e s}}^{\mathbf{e x t}} \cdot \mathrm{d} \mathbf{r}_{\mathbf{c m}} & =\int_{\Gamma_{\mathrm{cm}}}\left(\mathbf{F}_{\text {contato }}+\mathbf{P}\right) \cdot \mathrm{d} \mathbf{r}_{\mathbf{c m}} \\
& =\int_{\Gamma_{\mathrm{cm}}} \mathbf{f}_{\mathbf{c}} \cdot \mathrm{d} \mathbf{r}_{\mathbf{c m}}=-f_{\mathrm{c}} d
\end{aligned}
$$

\footnotetext{
${ }^{5}$ Ao escrever esse trecho do artigo, lembrei-me das seguintes palavras de Ivan S. Oliveira, pesquisador titular do Centro Brasileiro de Pesquisas Físicas, em seu livro Física Moderna para iniciados, interessados e aficionados [2] (volume 1, página 144): "Em física é assim: tratamos com pompa e detalhes matemáticos o que conhecemos; ao que não conhecemos associamos uma letra qualquer, damos um nome pomposo, e incluímos nos cálculos. É bárbaro!" O autor está se referindo ao conceito de massa efetiva, mas a citação reflete um pouco do espírito do que estamos fazendo aqui, introduzindo a igualdade 17 .
}

para um deslocamento $d$ do centro de massa do bloco. Com isso, obtemos:

$$
W_{\text {total }}=-f_{\mathrm{c}} d+\Delta T_{\text {rel }} .
$$

Substituindo então (16), 17) e 20 em (15), obtemos

$$
W_{\text {contato }}=-f_{\mathrm{c}} d+\Delta T_{\text {rel }}+\Delta U_{\text {int }} .
$$

Podemos definir a energia interna, $E_{\text {int }}$, do bloco da Fig. 1 como a soma das energias $T_{\text {rel }}, U_{\text {int }}$, e qualquer outra forma de energia que possa integrar o "conteúdo energético interno" desse bloco. Desde que apenas $T_{\text {rel }}$ e $U_{\text {int }}$ variem no processo de deslizamento horizontal do bloco, temos $\Delta E_{\text {int }}=\Delta T_{\text {rel }}+\Delta U_{\text {int }}$, e, portanto, podemos reescrever a última igualdade como

$$
W_{\text {contato }}=-f_{\mathrm{c}} d+\Delta E_{\text {int }} .
$$

Chegamos, então, a uma expressão para $W_{\text {contato }}$ - o trabalho total realizado pelas forças que o piso exerce sobre partículas do bloco da Fig. 1. ao longo de um deslocamento $d$ de seu centro de massa.

Considero inadequado - ou mesmo incorreto - chamar esse trabalho de trabalho da força de atrito cinético, porque não se trata de um trabalho de uma única força - nem mesmo da força resultante. Mas penso que é adequado chamar tal trabalho de trabalho do atrito cinético (sem a palavra "força"), porque é através do fenômeno de atrito entre as superfícies que esse trabalho é realizado - embora cada força que o piso exerce sobre uma partícula do bloco possa atuar, na realização do trabalho, não apenas na direção do movimento do centro de massa. Com isso, podemos reescrever a igualdade 22 como

$$
W_{\text {atrito cinético }}=-f_{\mathrm{c}} d+\Delta E_{\text {int }} \text {. }
$$

Então, insisto: ao olhar para a igualdade (23), não a leia como "trabalho da força de atrito cinético", mas como "trabalho do atrito cinético". Trata-se do trabalho total realizado pelas forças que o piso exerce sobre partículas do bloco da Fig.1. devido ao fenômeno de atrito cinético, ao longo de um deslocamento d de seu centro de massa.

A igualdade (23) não deve ser usada às cegas. Seu processo de obtenção deve ser bem compreendido, para que ela possa ser adaptada a problemas diferentes do apresentado, se alguma modificação se fizer necessária. Vamos analisar algumas variações possíveis do problema relativo à Fig.1. Na primeira, considere que o bloco da Fig.11é puxado horizontalmente para a direita através de uma força de tração constante de módulo $T$ (veja Fig. 2). Dependendo do valor de $T\left(T>f_{\mathrm{c}}, T<f_{\mathrm{c}}\right.$ ou $\left.T=f_{\mathrm{c}}\right)$, o bloco será acelerado, desacelerado ou irá se mover com velocidade constante. Refazendo os passos que levaram à igualdade (23), podemos facilmente identificar que a igualdade 15) deve ser modificada para

$$
W_{\text {contato }}=W_{\text {total }}-W_{T}-W_{\text {pesos }}-W_{\mathrm{F}_{\text {internas }}},
$$

com $W_{\text {pesos }}$ e $W_{\mathrm{F}_{\text {internas }}}$ ainda dados pelas igualdades 16 e (17), respectivamente, e com

$$
W_{T}=T d .
$$




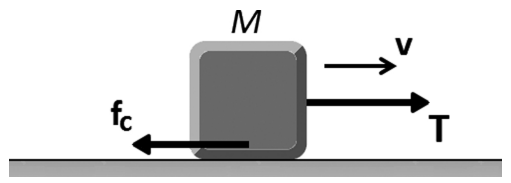

Figura 2: Variação do problema relativo à Fig. 1. bloco sendo puxado para a direita com tração $T$ constante.

Para o cálculo de $W_{\text {total }}$, devemos voltar às igualdades (19), e observar que devemos modificá-las para

$$
\begin{aligned}
\int_{\Gamma_{\mathrm{cm}}} \mathbf{F}_{\mathbf{r e s}}^{\mathbf{e x t}} \cdot \mathrm{d} \mathbf{r}_{\mathbf{c m}} & =\int_{\Gamma_{\mathrm{cm}}}\left(\mathbf{F}_{\text {contato }}+\mathbf{P}+\mathbf{T}\right) \cdot \mathrm{d} \mathbf{r}_{\mathbf{c m}} \\
& =\int_{\Gamma_{\mathrm{cm}}} \mathbf{f}_{\mathbf{c}} \cdot \mathrm{d} \mathbf{r}_{\mathbf{c m}}+\int_{\Gamma_{\mathrm{cm}}} \mathbf{T} \cdot \mathrm{d} \mathbf{r}_{\mathbf{c m}} \\
& =-f_{\mathrm{c}} d+T d
\end{aligned}
$$

A igualdade 20) (que resultou da substituição de 19 em (18) deve ser então modificada para

$$
W_{\text {total }}=-f_{\mathrm{c}} d+T d+\Delta T_{\text {rel }}
$$

Substituindo então (16), (17), 25) e (27) em (24), obtemos:

$$
\begin{aligned}
W_{\text {contato }} & =-f_{\mathrm{c}} d+T_{d}+\Delta T_{\text {rel }}-T_{d} d+\Delta U_{\text {int }} \\
& =-f_{\mathrm{c}} d+\Delta T_{\text {rel }}+\Delta U_{\text {int }},
\end{aligned}
$$

que é o mesmo resultado que o apresentado em (21). Segue então o mesmo desenvolvimento apresentado logo após a igualdade (21) - o que nos leva à igualdade (23). Ou seja, a igualdade 23 também se aplica a essa variação do problema inicial (mas isso não significa que o valor de $\Delta E_{\text {int }}$ seja o mesmo nos dois casos).

É fácil concluir (proponho que você verifique) que chegamos ao mesmo resultado (igualdade (23)) com uma tração horizontal $\mathbf{T}$ de módulo variável, podendo haver até mudança no sentido de $\mathbf{T}$ - com a condição, é claro, que a distância $d$ seja percorrida pelo bloco. Ainda temos, nesse caso, um cancelamento como o apresentado em 28, mas com $\int_{0}^{d} T_{x}(x) \mathrm{d} x$ no lugar de $T d$, sendo $T_{x}$ a componente $x$ de $\mathbf{T}$ (que pode ser positiva, negativa ou nula, para um dado $x){ }^{6}$

Vamos analisar mais uma variação do problema relativo à Fig.1. Desta vez, consideremos que o bloco está sobre uma esteira que se move aceleradamente para a esquerda (veja Fig. 3). Consideremos que a aceleração da esteira é tal que a força necessária para mover o bloco sem que ele deslize sobre ela tem módulo superior ao módulo

\footnotetext{
${ }^{6}$ Estamos, por enquanto, restringindo a direção de $\mathbf{T}$ à horizontal porque uma componente vertical variável de $\mathbf{T}$ alteraria o módulo da força normal $\mathbf{f}_{\mathrm{n}}$ sobre o bloco ao longo do percurso, e, com isso, $f_{\mathrm{c}}$ não seria mais constante (afinal, $f_{\mathrm{c}}=\mu_{\mathrm{c}} f_{\mathrm{n}}$, em que $\mu_{\mathrm{c}}$ é o coeficiente de atrito cinético entre as duas superfícies em contato); portanto, a igualdade 23 não seria mais válida. Todavia, observe que continuamos obtendo a igualdade 23 substituindo, na Fig.2 T por uma tração não horizontal, desde que sua componente vertical seja constante.
}

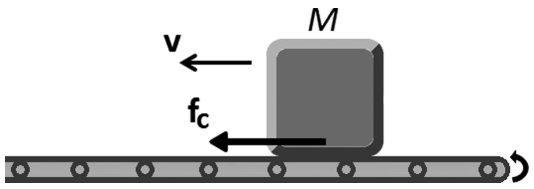

Figura 3: Variação do problema relativo à Fig.1 1 bloco sendo puxado para a esquerda por uma esteira acelerada, através da força de atrito cinético $\mathbf{f}_{\mathbf{c}}$.

máximo da força de atrito estático. Com isso, o bloco é movido para a esquerda pela forca de atrito cinético, $\mathbf{f}_{\mathrm{c}}$, exercida pela esteira. Isso significa, é claro, que há movimento relativo entre o bloco e a esteira (ou não haveria atrito cinético), mas o bloco é puxado para a esquerda, no referencial do laboratório. O que temos de especial nessa situação é que $\mathbf{f}_{\mathbf{c}}$ tem o mesmo sentido do deslocamento vetorial $\mathbf{d}$ sofrido pelo bloco (no referencial do laboratório), e, portanto, temos $\int_{\Gamma_{\mathbf{c m}}} \mathbf{f}_{\mathbf{c}} \cdot \mathrm{d} \mathbf{r}_{\mathbf{c m}}=f_{\mathrm{c}} d$ (em vez de $\left.-f_{\mathrm{c}} d\right)$. No mais, as contas são idênticas às já realizadas, e obtemos:

$$
W_{\text {atrito cinético }}=f_{\mathrm{c}} d+\Delta E_{\mathrm{int}} .
$$

É interessante perceber que o termo $f_{\mathrm{c}} d$ em 29 independe do valor da velocidade relativa entre o bloco e a esteira; o que importa é que a esteira tenha aceleração suficiente para que o bloco não possa ser arrastado por atrito estático. E quanto ao valor do termo $\Delta E_{\text {int }}$, em (29)? Ele muda com o aumento dessa velocidade relativa? Vejamos. Imagine vários blocos idênticos ao da Fig. 3 sofrendo o mesmo deslocamento $d$ no referencial do laboratório, mas com deslocamentos diferentes em relação à esteira. Em princípio (aqui é minha intuição física se expressando, e ela pode não estar correta), quanto maior a velocidade relativa entre o bloco e a esteira, maior o aquecimento do bloco; portanto, maior o aumento de sua energia interna, ao final do percurso de comprimento $d$ no referencial do laboratório. Assim, o valor de $\Delta E_{\text {int }}$, em (29), não pode ser extraído apenas da análise do movimento do centro de massa do bloco. Certamente isso dificulta a obtenção do valor de $W_{\text {atrito cinético }} \mathrm{em}$ (29) (se tivermos interesse nele), mas a análise do balanço energético do sistema não estaria completa sem o termo $\Delta E_{\text {int }}$. (Contudo, se desejarmos modelar o bloco como uma partícula - ou seja, se estivermos interessados apenas no movimento de seu centro de massa -, não precisaremos incluir $\Delta E_{\mathrm{int}}$ explicitamente na análise do balanço energético, conforme veremos na próxima seção.)

Observe que podemos reunir os resultados 23) e 29) em uma única igualdade, escrevendo $\mathbf{f}_{\mathbf{c}} \cdot \mathbf{d}$ no lugar de $-f_{\mathrm{c}} d$ e $f_{\mathrm{c}} d$, respectivamente, obtendo:

$$
W_{\text {atrito cinético }}=\mathbf{f}_{\mathbf{c}} \cdot \mathbf{d}+\Delta E_{\text {int }} .
$$

Esta é a equação mais geral, até este ponto, para o cálculo de $W_{\text {atritocinético (válida para os problemas }}$ relativos às Figs. 1. 2 e 3), mas podemos torná-la ainda mais geral. Esteja atento(a) às seguintes considerações: 
- Vamos manter a restrição de que o bloco que sofre a ação do atrito cinético se move, no referencial do laboratório, sempre com velocidade tangente à superfície com a qual faz contato, de forma que $\int_{\Gamma_{\mathrm{cm}}} \mathbf{f}_{\mathbf{n}} \cdot \mathrm{d} \mathbf{r}_{\mathbf{c m}}=0, \mathrm{e}$, portanto, $\int_{\Gamma_{\mathrm{cm}}} \mathbf{F}_{\mathbf{c o n t a t o}} \cdot \mathrm{d} \mathbf{r}_{\mathbf{c m}}=$ $\int_{\Gamma_{\mathrm{cm}}} \mathbf{f}_{\mathrm{c}} \cdot \mathrm{d} \mathbf{r}_{\mathbf{c m}}$.

- Consideremos também que todas as outras forças externas, além das forças de contato (associadas ao atrito cinético) sobre o bloco, são tais que a soma dos trabalhos realizados por elas, $W_{\mathrm{F}_{\text {externas }} \text { outras }}$, pode ser expressa na forma $\int_{\Gamma_{\mathrm{cm}}} \mathbf{F}_{\text {outras }}^{\mathbf{e x t}} \cdot \mathrm{d} \mathbf{r}_{\mathbf{c m}}$, em que $\mathbf{F}_{\text {outras }}^{\text {ext }}$ é a soma de tais forças.7

- Vamos continuar considerando que as forças internas são tais que podemos continuar escrevendo $W_{\mathrm{F}_{\text {internas }}}=-\Delta U_{\text {int }}$.

- $\mathrm{E}$ vamos manter a igualdade $\Delta E_{\text {int }}=\Delta T_{\text {rel }}+$ $\Delta U_{\text {int }}$.

Com isso, temos:

$$
\begin{aligned}
W_{\text {total }} & =W_{\text {contato }}+W_{\mathrm{F}_{\text {externas outras }}}+W_{\mathrm{F}_{\text {internas }}} \Rightarrow \\
W_{\text {contato }} & =W_{\text {total }}-W_{\mathrm{F}_{\text {externas outras }}}-\underbrace{W_{\mathrm{F}_{\text {internas }}}}_{-\Delta U_{\text {int }}}
\end{aligned}
$$

em que

$$
\begin{aligned}
W_{\text {total }} & =\int_{\Gamma_{\mathrm{cm}}} \mathbf{F}_{\mathbf{r e s}}^{\mathbf{e x t}} \cdot \mathrm{d} \mathbf{r}_{\mathbf{c m}}+\Delta T_{\mathrm{rel}} \\
& =\underbrace{\int_{\Gamma_{\mathrm{cm}}} \mathbf{F}_{\mathbf{c o n t a t o}} \cdot \mathrm{d} \mathbf{r}_{\mathbf{c m}}}_{\int_{\Gamma_{\mathrm{cm}}} \mathbf{f}_{\mathbf{c}^{\cdot}} \mathrm{d} \mathbf{r}_{\mathrm{cm}}}+\int_{\Gamma_{\mathrm{cm}}} \mathbf{F}_{\text {outras }}^{\mathbf{e x t}} \cdot \mathrm{d} \mathbf{r}_{\mathbf{c m}} \\
& +\Delta T_{\mathrm{rel}} \cdot
\end{aligned}
$$

Substituindo 32 em 31, obtemos:

$$
\begin{aligned}
W_{\text {contato }} & =\int_{\Gamma_{\mathrm{cm}}} \mathbf{f}_{\mathbf{c}} \cdot \mathrm{d} \mathbf{r}_{\mathbf{c m}}+\int_{\Gamma_{\mathrm{cm}}}^{\mathbf{F}_{\text {outras }}^{\text {ext }} \cdot \mathrm{d} \mathbf{r}_{\mathbf{c m}}} \\
& -W_{\text {Fexternasoutras }_{\text {ext }}}^{\Delta T_{\text {rel }}+\Delta U_{\text {int }}},
\end{aligned}
$$

o que nos leva (agora trocando " $W_{\text {contato" por }}$ " $\left.W_{\text {atrito cinético }}\right)$ a

$$
W_{\text {atrito cinético }}=\int_{\Gamma_{\mathrm{cm}}} \mathbf{f}_{\mathbf{c}} \cdot \mathrm{d} \mathbf{r}_{\mathbf{c m}}+\Delta E_{\text {int }} .
$$

A vantagem desta expressão para $W_{\text {atrito cinético, em rela- }}$ ção à expressão em (30), é que em (34) podemos trabalhar com $\mathbf{f}_{\mathbf{c}}$ com módulo e sentido variáveis. $\mathrm{O}$ módulo de $\mathbf{f}_{\mathbf{c}}$ pode mudar devido à mudança do coeficiente de atrito entre as superfícies em contato, ou do módulo da força normal $\mathbf{f}_{\mathbf{n}}$, ao longo do percurso do bloco. O desenvolvimento de (31) a (34), com as considerações que o precedem, resume e amplia todo o desenvolvimento anterior nesta seção.

${ }^{7}$ Esta é uma restrição à qual devemos estar particularmente atentos, na aplicação da equação 34 , obtida logo adiante.
Em seu trabalho, Nelson e Carneiro Filho obtiveram a igualdade

$$
W_{a}=-f_{a} d+\Delta E_{\text {int }} \text { (igualdade (18) da ref. 1), }
$$

que corresponde à igualdade 23) deste artigo, com " $W_{a}$ " no lugar de " $W_{\text {atrito cinético }} \mathrm{e}^{\text {" }} f_{a}$ " no lugar de " $f_{\mathrm{c}}$ ". No caminho para a obtenção daquela igualdade, o teorema trabalho - energia cinética para um sistema de partículas foi demonstrado para um sistema de apenas duas partículas, enquanto aqui foi apresentada a demonstração completa para um sistema de $N$ partículas. Tudo bem; foi uma opção dos autores, deixando o texto bastante conciso e acessível a uma gama maior de estudantes do início de um curso de graduação em física ou área afim - embora, talvez, deixando de satisfazer os interesses de estudantes que buscam maior rigor. Mas não é nesse ponto que reside a crítica feita aqui àquele trabalho. Segundo Nelson e Carneiro Filho, a expressão correta para o trabalho realizado pela força de atrito cinético, em um problema como aquele relativo à Fig.11. equivale à apresentada na igualdade (23) deste artigo; ou seja, além de os autores não admitirem a possibilidade de modelarmos como uma partícula um bloco como o da Fig. 1. para o cálculo do trabalho realizado pela força de atrito cinético, usam, equivocadamente (e em várias partes do texto) a expressão "trabalho da força de atrito" - que, ironicamente (conforme discutimos no início desta seção) é inadequada quando modelamos o corpo como um sistema da partículas.

Veremos, na próxima seção, que podemos, dentro dos rigores da mecânica clássica, modelar o bloco da Fig.11 (e também os blocos das Figs. 2 e 3) como uma partícula, e dar significado (útil) à expressão trabalho realizado pela força (aqui sim, convém usar esta palavra) de atrito cinético.

\section{Modelando o bloco da Fig. 1 como uma partícula}

Já temos o que precisamos para modelar o bloco da Fig.11 como uma partícula e dar significado à expressão "trabalho realizado pela força de atrito cinético" (ao longo de um deslocamento $d$ sofrido pelo bloco). Trata-se da igualdade (9). Vou reescrevê-la aqui, dando-lhe destaque:

$$
\int_{\Gamma_{\mathrm{cm}}} \mathbf{F}_{\mathbf{r e s}}^{\mathbf{e x t}} \cdot \mathrm{d} \mathbf{r}_{\mathbf{c m}}=\Delta\left(\frac{1}{2} M v_{\mathrm{cm}}^{2}\right) \cdot
$$

Podemos pensar essa igualdade como a expressão matemática de um terceiro teorema trabalho - energia cinética. Segundo tal teorema, o trabalho da força externa resultante, aplicada ao centro de massa de um sistema de partículas, é igual à variação da energia cinética de translação do sistema (que pode ser denominada "energia cinética do centro de massa"). Trata-se de um teorema não para uma partícula, nem para um sistema de par- 
tículas, mas para o centro de massa de um sistema de partículas 8

Em geral, podemos separar a integral em 35 em dois ou mais termos, dependendo não apenas da composição da força externa resultante, mas também da forma como desejamos agrupar tais forças. Assim, no que se refere ao problema relativo à Fig. 1, podemos extrair do primeiro membro da igualdade 350 o termo $\int_{\Gamma_{\mathrm{cm}}} \mathbf{f}_{\mathbf{c}} \cdot \mathrm{d} \mathbf{r}_{\mathbf{c m}}$ e pensálo como o trabalho realizado pela força de atrito cinético - aplicada ao centro de massa do sistema. Sabemos que $\mathbf{f}_{\mathbf{c}}$ não é uma força simples, mas uma componente da força resultante $\mathbf{F}_{\text {contato }}$. Contudo, quando aplicada ao centro de massa de um sistema de partículas, podemos tratá-la como uma força simples, porque a integração em $\int_{\Gamma_{\mathrm{cm}}} \mathbf{f}_{\mathbf{c}} \cdot \mathrm{d} \mathbf{r}_{\mathbf{c m}}$ se dá ao longo de um caminho único: o do centro de massa do sistema. Logo, faz sentido modelarmos um sistema como o bloco da Fig.1 como uma partícula e definirmos o trabalho realizado pela força de atrito cinético, ao longo de um determinado percurso $\Gamma_{\mathrm{cm}}$ do centro de massa do sistema, como

$$
W_{f_{\mathrm{c}}}=\int_{\Gamma_{\mathrm{cm}}} \mathbf{f}_{\mathbf{c}} \cdot \mathrm{d} \mathbf{r}_{\mathbf{c m}},
$$

que no caso do problema relativo à Fig.1 1 leva a

$$
W_{f_{\mathrm{c}}}=-f_{\mathrm{c}} d
$$

que é a igualdade cujo uso é criticado por Nelson e Carneiro Filho [1] ${ }^{9}$

Vemos, assim, que, no que se refere ao cálculo do trabalho de uma força, a igualdade (35) nos permite retomar o conceito de partícula única quando inicialmente optamos por modelar o sistema físico como um sistema de partículas. Portanto, mesmo modelando o bloco da Fig. 1] como um sistema de partículas - e, desse modo, criando espaço para grandezas como temperatura e energia interna podemos retomar o conceito de partícula voltando nossa atenção ao centro de massa do sistema. Não precisamos, então, abrir mão da ideia de que o atrito cinético produz aquecimento do bloco da Fig.11 e, ao mesmo tempo, podemos modelá-lo como uma partícula: basta concentrarmos nossa atenção em seu centro de massa. Mas é claro que, quando o fazemos, usando a igualdade (37) ou a igualdade (36) (que é mais geral que a anterior), o termo $E_{\text {int }}$ não aparece nas contas. Podemos manter a ideia de que o atrito cinético produz aquecimento do bloco da Fig. 1] porque sabemos que estamos concentrando nossa atenção no centro de massa do sistema, sem nos esquecermos do sistema como um todo. E concentrando nossa atenção no centro de massa, podemos resolver problemas como os apresentados a seguir.

${ }^{8}$ Nem todos os livros de física básica apresentam esse teorema. Você irá encontrá-lo, por exemplo, no livro de Tipler e Mosca [3] - mais especificamente na seção 6.5, intitulada "Trabalho no Centro de Massa".

${ }^{9}$ Compare as igualdades 36 e 37 respectivamente às igualdades (34) e 23. Lembre-se de que critiquei, para as igualdades (34) e 23, o uso da expressão "trabalho da força de atrito cinético", e por isso usei nas mesmas " $W_{\text {atrito cinético }}$ ", em vez de " $W_{f_{\mathrm{c}}}$ ".
Se desejamos, por exemplo, prever a distância $d$ que será percorrida pelo bloco da Fig. 1. estando ele com velocidade inicial de módulo $v$, podemos aplicar ao mesmo o teorema expresso pela igualdade (35), obtendo:

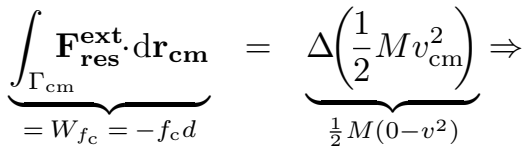

$$
\begin{aligned}
& d=\frac{M v^{2}}{2 f_{\mathrm{c}}}=\frac{v^{2}}{2 \mu_{\mathrm{c}} g},
\end{aligned}
$$

onde usamos, na última igualdade, $f_{\mathrm{c}}=\mu_{\mathrm{c}} f_{\mathrm{n}}=\mu_{\mathrm{c}} M g$. Também podemos obter esse resultado a partir da aplicação da segunda lei de Newton ao bloco, mas o que importa, para o argumento que apresento, é que a igualdade (37) nos permitiu obter o resultado correto para o problema proposto. O uso da igualdade (23), em vez da igualdade (37), não leva a nada diferente, na resolução deste problema: partindo da igualdade $(6)$ e substituindo na mesma $W_{\text {total }}$ por $W_{\text {atrito cinético }}+W_{\mathrm{F}_{\text {internas }}}$, $\operatorname{com} W_{\text {atrito cinético }}=-f_{\mathrm{c}} d+\Delta E_{\text {int }} \mathrm{e} W_{\mathrm{F}_{\text {internas }}}=-\Delta U_{\text {int }}$, obtemos uma igualdade equivalente à igualdade (38), porque o termo $\Delta E_{\text {int }}$ é cancelado conjuntamente com os termos $\Delta T_{\text {rel }}$ e $\Delta U_{\text {int }}$.

Uma interessante variação desse problema: temos, na Fig. 4. uma pista em forma de "U", sendo que só há atrito entre o bloco e a pista na base horizontal da mesma. Considerando a condição inicial expressa na figura, e desprezando as dimensões do bloco, em comparação com a largura $L$ da base da pista (a figura estaria fora de escala), qual será a posição final do bloco, em relação ao ponto $A$ ? Novamente, podemos aplicar ao bloco o teorema expresso pela igualdade $(35)$, considerando que o estado inicial é o indicado na Fig. 4 e que o estado final é aquele em que o bloco está em repouso. Cada vez que o bloco sobe e desce uma das rampas, a contribuição de seu peso para a integral no membro esquerdo de (35) se anula, de forma que, ao final, temos $\int_{\Gamma_{\mathrm{cm}}} \mathbf{F}_{\text {res }}^{\mathbf{e x t}} \cdot \mathrm{d} \mathbf{r}_{\mathbf{c m}}=$ $W_{f_{\mathrm{c}}}=\int_{\Gamma_{\mathrm{cm}}} \mathbf{f}_{\mathbf{c}} \cdot \mathrm{d} \mathbf{r}_{\mathbf{c m}}=-f_{\mathrm{c}} D$, sendo $D$ a distância total percorrida pelo bloco na base da pista. Com isso, a igualdade 35 nos dá $-f_{\mathrm{c}} D=\frac{1}{2} M\left(0-v^{2}\right)$, e, portanto, $D=\frac{M v^{2}}{2 f_{\mathrm{c}}}=\frac{v^{2}}{2 \mu_{\mathrm{c}} g}$. Para obtermos a posição final do bloco, devemos dividir $D$ por $L$ e subtrair do resultado sua parte inteira, que denotarei por $p i(D / L)$, obtendo então a parte decimal da divisão, $p d(D / L)$. Se $p i(D / L)$ é par, então a posição final do bloco (medida a partir do ponto $A)$ é $x_{f}=L \times p d(D / L)$; do contrário, é $x_{f}=$ $L-L \times p d(D / L)=L(1-p d(D / L))$. O uso da igualdade

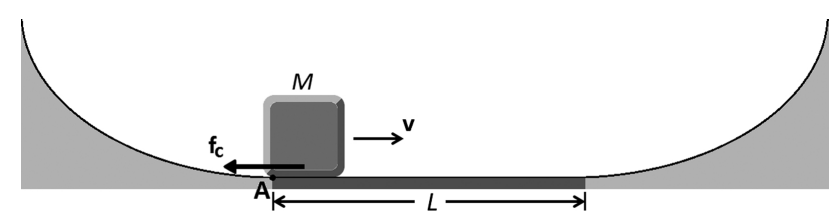

Figura 4: Bloco se movendo em uma pista em forma de "U". Só há atrito entre o bloco e a pista na base horizontal da mesma. 
36), associada à equação (35), nos permitiu obter o resultado correto para o problema proposto, mostrando que o uso daquela igualdade se justifica.

\section{Conclusão e comentários adicionais}

Contrariando o que afirmaram Nelson e Carneiro Filho no artigo intitulado "A utilização equivocada do conceito de partícula no cálculo do trabalho da força de atrito" [1], neste texto vimos que podemos, dentro dos rigores da mecânica clássica, modelar um bloco como o da Fig. 1 não apenas como um sistema de partículas, mas também como uma partícula, e dar significado (útil) à expressão "trabalho realizado pela força de atrito cinético". Em tal modelagem, o uso da igualdade (37), ou similar, apontado pelos autores da referência 1 como equivocado e "desprovido de qualquer justificativa", é legítimo. Podemos usar tal igualdade (ou similar), conjuntamente com a igualdade (35), na resolução de problemas como os apresentados na parte final da seção 4, nos quais o que nos interessa é o movimento do centro de massa do sistema - sem nenhuma contradição com o fato de que o atrito cinético produz aquecimento dos sistemas físicos em contato, porque as igualdades 36 e $(37)$ nos fornecem o trabalho realizado pela força de atrito cinético aplicada ao centro de massa do sistema de partículas sob consideração.

No caso da modelagem do bloco como um sistema de partículas - que, fique claro, não constitui um retrato fiel da realidade, mas outra opção de modelagem -, argumentei que a expressão "trabalho da força de atrito cinético" é inadequada, porque a força de atrito cinético, $\mathbf{f}_{\mathbf{c}}$, não constitui uma força única, mas uma soma de forças - ou, mais especificamente, a componente paralela ao plano de contato da soma de forças $\mathbf{F}_{\text {contato }} 10$ Propus, então, a expressão "trabalho do atrito cinético", e as igualdades (23) e (34) devem ser usadas quando temos interesse em incluir, nos cálculos, a variação da energia interna do sistema, $\Delta E_{\text {int }}$, devido ao atrito cinético. Um exemplo interessante está disponibilizado online como Material Suplementar. Lembre-se: $W_{\text {atrito cinético é o tra- }}$ balho total realizado pelas forças que o piso (ou outra superfície) exerce sobre o corpo, devido ao fenômeno de atrito cinético, ao longo de um determinado percurso de seu centro de massa. E esteja atento(a) às considerações que precederam o desenvolvimento da igualdade (31) à igualdade (34), para aplicar a igualdade 34 com segurança.

Esteve sempre presente, na elaboração deste artigo, meu desejo de mostrar - especialmente a um(a) estudante de física no início de sua formação, e mesmo que apenas para um problema específico - como o processo de modelagem desempenha um papel fundamental no desenvolvimento dessa ciência. Nesse sentido, determinadas "certezas" devem ser evitadas, e, no final, a física funciona quando consegue harmonizar teoria e experimentos (ou observações).

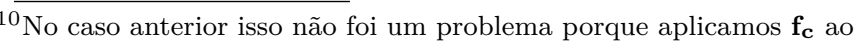
centro de massa do sistema de partículas.
}

Não posso deixar de comentar que o tema "atrito" ainda envolve muita investigação científica, e em um nível de sofisticação bastante superior às modelagens que foram discutidas neste artigo. Como apontam Alarcón e colaboradores [4], esforços em micro e nanoescalas e na biologia, durante as últimas décadas, têm resultado no ressurgimento de atividades na área de tribologia. ${ }^{11} \mathrm{Se}$ for de seu interesse, veja, por exemplo, as referências 5 a 15, na publicação de Alarcón e colaboradores, e artigos relacionados. Tais trabalhos envolvem tratamentos teóricos avançados (que vão muito além da fenomenologia), microscopia de força atômica e investigações com nanotubos de carbono e folhas de grafeno, entre outras coisas. No entanto, como comentam Alarcón e colaboradores [4], é notável que embora fenômenos complexos não-lineares possam ser observados em escalas micrométricas, é frequentemente o caso que em escalas de maior comprimento as chamadas leis de Amontons-Coulomb simples (leis AC) com as quais estamos tão familiarizados (como $f_{\mathrm{c}}=\mu_{\mathrm{c}} f_{\mathrm{n}}$, incluindo a independência do valor de $f_{\mathrm{c}}$ em relação à velocidade relativa entre as superfícies em contato) são válidas. Nas modelagens apresentadas neste trabalho, nos restringimos a corpos de dimensões macroscópicas, com a suposição da validade das leis AC, e fizemos uso da mecânica newtoniana, apenas.

\section{Agradecimento}

Agradeço a Adonias Barros, Eduardo Silva e Pedro Júnior - todos do Curso de Física-Licenciatura do Núcleo de Formação Docente da UFPE - pelas discussões realizadas na fase inicial deste trabalho. Agradecimento adicional a Adonias, que teve a iniciativa de checar todas as contas do artigo.

\section{Material suplementar}

O seguinte material suplementar está disponível online: Combinando intuição e rigor.

\section{Referências}

[1] O.R. Nelson e R.C. Filho, Rev. Bras. Ens. Fis. 33, 2308 (2011).

[2] I.S. Oliveira, Física Moderna para iniciados, interessados e aficionados (Editora Livraria da Física, São Paulo, 2005), v. 1.

[3] P.A. Tipler e G. Mosca, Fúsica para Cientistas e Engenheiros (LTC, Rio de Janeiro, 2013), v. 1, $6^{\text {a }}$ ed.

[4] H. Alarcón, T. Salez, C. Poulard, J.F. Bloch, E. Raphaël, K. Dalnoki-Veress e F. Restagno, Physical Review Letters 116, 015502 (2016).

\footnotetext{
${ }^{11}$ Ciência e tecnologia da interação de superfícies em movimento relativo e assuntos e práticas relacionados.
} 